Supplement of Hydrol. Earth Syst. Sci., 22, 2163-2185, 2018

https://doi.org/10.5194/hess-22-2163-2018-supplement

(C) Author(s) 2018. This work is distributed under

the Creative Commons Attribution 3.0 License.

(c) (1)

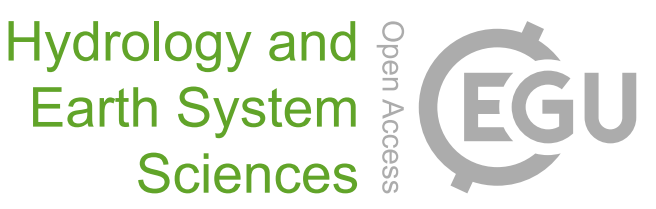

Supplement of

\title{
Are we using the right fuel to drive hydrological models? A climate impact study in the Upper Blue Nile
}

Stefan Liersch et al.

Correspondence to: Stefan Liersch (liersch@pik-potsdam.de)

The copyright of individual parts of the supplement might differ from the CC BY 3.0 License. 
Table S 1: Regional Climate Models (RCM) and driving Earth System Models (ESM)

\begin{tabular}{l|cccc}
\hline Driving ESM & \multicolumn{4}{|c}{ RCM } \\
\hline CanESM2 & SMHI-RCA4 & CanRCM4 & KNMI-RACMO22T & DMI-HIRHAM4 \\
CNRM-CM5 & $\mathrm{x}$ & $\mathrm{x}$ & & \\
EC-EARTH & $\mathrm{x}$ & & $\mathrm{x}$ & $\mathrm{x}$ \\
GFDL-ESM2M & $\mathrm{x}$ & & & \\
MIROC5 & $\mathrm{x}$ & & & \\
MPI-ESM-LR & $\mathrm{x}$ & & & \\
NorESM1-M & $\mathrm{x}$ & & & \\
\hline
\end{tabular}

Table S 2: Model IDs

\begin{aligned} & \hline ID Model \\ & \hline 1 GFDL \\ & 2 HadGEM \\ & 3 IPSL \\ & 4 MIROC \\ & 5 NorESM \\ & 6 CanESM2-RCM4 \\ & 7 CanESM2-RCA4 \\ & 8 CNRM-CM5-RCA4 \\ & 9 GFDL-RCA4 \\ & 10 EC-EARTH-Hirham5 \\ & 11 EC-EARTH-RACMO \\ & 12 EC-EARTH-RCA4 \\ & 13 MIROC-RCA4 \\ & 14 MPI-M-ESM-LR-RCA4 \\ & 15 NorESM1-RCA4 \\ & \hline\end{aligned}


Table S 3: Daily precipitation parameters in [mm] in the period 1970-1999

\begin{tabular}{|c|c|c|c|c|c|c|}
\hline Model & nDays $>1 \mathrm{~mm}$ & ave & $\max$ & SD & ave (JAS) & $\mathrm{SD}(\mathrm{JAS})$ \\
\hline WATCH Forcing Data (WFD) & 164.1 & 3.9 & 63.4 & 6.9 & 9.1 & 9.6 \\
\hline \multicolumn{7}{|c|}{ Uncorrected (UC) model runs } \\
\hline GFDL & 188.4 & 3.8 & 49.5 & 4.7 & 7.6 & 3.8 \\
\hline HadGEM & 228.1 & 3.6 & 21.9 & 3.6 & 7.5 & 2.8 \\
\hline IPSL & 134.4 & 1.7 & 28.4 & 2.8 & 5.2 & 3.2 \\
\hline MIROC & 194.2 & 3.7 & 66.2 & 5.5 & 7.8 & 7.3 \\
\hline NorESM & 204.8 & 3.5 & 28.5 & 4.5 & 5.4 & 4.1 \\
\hline CanESM2-RCM4 & 250.1 & 4.4 & 57.7 & 4.4 & 8.6 & 3.7 \\
\hline CanESM2-RCA4 & 130.4 & 1.8 & 39.6 & 3.1 & 5.3 & 3.9 \\
\hline CNRM-CM5-RCA4 & 180.6 & 2.6 & 30.3 & 3.4 & 6.5 & 3.2 \\
\hline GFDL-RCA4 & 183.8 & 3.4 & 40.4 & 4.5 & 8.5 & 4.0 \\
\hline EC-EARTH-Hirham5 & 177.8 & 3.7 & 40.7 & 5.2 & 9.0 & 5.5 \\
\hline EC-EARTH-RACMO & 213.6 & 3.8 & 52.3 & 4.5 & 7.8 & 4.7 \\
\hline EC-EARTH-RCA4 & 197.4 & 4.2 & 44.9 & 5.3 & 10.1 & 4.5 \\
\hline MIROC-RCA4 & 191.8 & 3.4 & 38.6 & 4.6 & 8.8 & 4.3 \\
\hline MPI-M-ESM-LR-RCA4 & 177.0 & 3.7 & 42.9 & 5.1 & 9.7 & 4.9 \\
\hline NorESM1-RCA4 & 157.3 & 2.9 & 41.0 & 4.4 & 8.1 & 5.0 \\
\hline \multicolumn{7}{|c|}{ Bias-corrected (BC) model runs } \\
\hline GFDL & 220.7 & 3.9 & 30.4 & 4.3 & 8.6 & 4.0 \\
\hline HadGEM & 180.7 & 3.9 & 73.5 & 5.2 & 8.4 & 4.9 \\
\hline IPSL & 153.1 & 3.7 & 65.8 & 6.5 & 8.9 & 6.8 \\
\hline MIROC & 192.9 & 3.9 & 90.8 & 6.4 & 8.7 & 9.1 \\
\hline NorESM & 187.1 & 3.9 & 51.0 & 5.5 & 8.5 & 6.4 \\
\hline CanESM2-RCM4 & 174.2 & 3.8 & 305.8 & 8.3 & 8.8 & 13.4 \\
\hline CanESM2-RCA4 & 130.4 & 3.2 & 142.0 & 7 & 8.6 & 10.5 \\
\hline CNRM-CM5-RCA4 & 161.6 & 3.7 & 117.3 & 6.9 & 8.5 & 9.4 \\
\hline GFDL-RCA4 & 159.8 & 3.9 & 133.6 & 7.6 & 9.2 & 10.8 \\
\hline EC-EARTH-Hirham5 & 159.6 & 4.0 & 85.1 & 7.3 & 8.8 & 10.5 \\
\hline EC-EARTH-RACMO & 155.1 & 3.8 & 210.8 & 8.8 & 9.0 & 13.7 \\
\hline EC-EARTH-RCA4 & 164.2 & 3.8 & 155.1 & 7.4 & 9.0 & 10.7 \\
\hline MIROC-RCA4 & 158.7 & 3.6 & 107.5 & 7.1 & 8.5 & 9.6 \\
\hline MPI-M-ESM-LR-RCA4 & 153.8 & 3.7 & 108.0 & 7.8 & 8.8 & 11.5 \\
\hline NorESM1-RCA4 & 146.2 & 3.5 & 144.5 & 7.5 & 8.9 & 11.2 \\
\hline
\end{tabular}


Table S 4: Daily precipitation parameters in [mm] in the period 1970-1999, absolute differences to WATCH Forcing Data (WFD)

\begin{tabular}{|c|c|c|c|c|c|c|}
\hline Model & nDays $>1 \mathrm{~mm}$ & ave & $\max$ & SD & ave (JAS) & $\mathrm{SD}(\mathrm{JAS})$ \\
\hline & \multicolumn{6}{|c|}{ Uncorrected (UC) model runs } \\
\hline GFDL & 24.3 & -0.1 & -13.9 & 0.7 & -1.5 & 0.4 \\
\hline HadGEM & 63.9 & -0.4 & -41.5 & 0.5 & -1.6 & 0.3 \\
\hline IPSL & -29.7 & -2.2 & -35 & 0.4 & -3.9 & 0.3 \\
\hline MIROC & 30.1 & -0.2 & 2.7 & 0.8 & -1.3 & 0.8 \\
\hline NorESM & 40.6 & -0.4 & -34.9 & 0.7 & -3.7 & 0.4 \\
\hline CanESM2-RCM4 & 86.0 & 0.5 & -5.8 & 0.6 & -0.5 & 0.4 \\
\hline CanESM2-RCA4 & -33.8 & -2.1 & -23.8 & 0.4 & -3.8 & 0.4 \\
\hline CNRM-CM5-RCA4 & 16.5 & -1.3 & -33.1 & 0.5 & -2.5 & 0.3 \\
\hline GFDL-RCA4 & 19.7 & -0.5 & -23.0 & 0.6 & -0.5 & 0.4 \\
\hline EC-EARTH-Hirham5 & 13.6 & -0.3 & -22.8 & 0.8 & -0.1 & 0.6 \\
\hline EC-EARTH-RACMO & 49.5 & -0.1 & -11.1 & 0.6 & -1.3 & 0.5 \\
\hline EC-EARTH-RCA4 & 33.3 & 0.3 & -18.6 & 0.8 & 1.0 & 0.5 \\
\hline MIROC-RCA4 & 27.6 & -0.5 & -24.9 & 0.7 & -0.2 & 0.4 \\
\hline MPI-M-ESM-LR-RCA4 & 12.9 & -0.2 & -20.6 & 0.7 & 0.6 & 0.5 \\
\hline \multirow[t]{2}{*}{ NorESM1-RCA4 } & -6.8 & -1.1 & -22.4 & 0.6 & -1.0 & 0.5 \\
\hline & \multicolumn{6}{|c|}{ Bias-corrected (BC) model runs } \\
\hline GFDL & 56.6 & 0.0 & -33 & 0.6 & -0.5 & 0.4 \\
\hline HadGEM & 16.6 & 0.0 & 10.1 & 0.7 & -0.7 & 0.5 \\
\hline IPSL & -11.0 & -0.2 & 2.4 & 0.9 & -0.2 & 0.7 \\
\hline MIROC & 28.8 & 0.0 & 27.4 & 0.9 & -0.4 & 1.0 \\
\hline NorESM & 22.9 & 0.0 & -12.4 & 0.8 & -0.6 & 0.7 \\
\hline CanESM2-RCM4 & 10.1 & -0.1 & 242.3 & 1.2 & -0.3 & 1.4 \\
\hline CanESM2-RCA4 & -33.7 & -0.7 & 78.6 & 1.0 & -0.5 & 1.1 \\
\hline CNRM-CM5-RCA4 & -2.5 & -0.2 & 53.8 & 1.0 & -0.6 & 1.0 \\
\hline GFDL-RCA4 & -4.4 & -0.1 & 70.2 & 1.1 & 0.1 & 1.1 \\
\hline EC-EARTH-Hirham5 & -4.6 & 0.1 & 21.7 & 1.1 & -0.3 & 1.1 \\
\hline EC-EARTH-RACMO & -9.1 & -0.1 & 147.3 & 1.3 & -0.1 & 1.4 \\
\hline EC-EARTH-RCA4 & 0.1 & -0.1 & 91.6 & 1.1 & -0.1 & 1.1 \\
\hline MIROC-RCA4 & -5.4 & -0.3 & 44.0 & 1.0 & -0.6 & 1.0 \\
\hline MPI-M-ESM-LR-RCA4 & -10.3 & -0.2 & 44.5 & 1.1 & -0.3 & 1.2 \\
\hline NorESM1-RCA4 & -17.9 & -0.4 & 81.0 & 1.1 & -0.1 & 1.2 \\
\hline
\end{tabular}

$n$ Days $>1 \mathrm{~mm}=$ Average number of days with precipitation $>1 \mathrm{~mm}$ per annum; ave $=$ average daily precipitation; $\max =$ maximum daily precipitation

$S D=$ standard deviation; ave $(J A S)=$ average precipitation in July, August, and September $S D(J A S)=$ standard deviation of daily precipitation in July, August, and September 
Table S 5: Relative deviation [\%] of Flow Duration Curve (FDC) values between uncorrected (UC) Earth System Models (ESMs) and WATCH Forcing Data (WFD) in the period 1970-1999. $Q_{p t}=$ percentile of discharge.

\begin{tabular}{rrrrrr}
$Q_{p t}$ & GFDL & HadGEM & IPSL & MIROC & NorESM \\
\hline 99.99 & 3.0 & 37.9 & -107.0 & -106.7 & -106.8 \\
99.9 & 3.3 & 55.9 & -106.6 & -103.0 & -106.2 \\
99 & -63.8 & -37.5 & -101.6 & -36.7 & -98.7 \\
95 & -37.0 & -21.8 & -100.4 & 15.2 & -38.2 \\
90 & 9.8 & -22.5 & -100.2 & 16.1 & -10.3 \\
\hline 80 & 49.1 & -26.8 & -100.1 & 5.1 & -7.4 \\
70 & 53.8 & -20.3 & -99.9 & 4.1 & 12.2 \\
60 & 49.5 & -19.2 & -98.5 & 1.8 & 14.6 \\
50 & 55.9 & -18.9 & -96.2 & 0.8 & 14.1 \\
40 & 67.1 & -17.9 & -94.6 & -1.1 & 4.1 \\
30 & 45.4 & -19.2 & -93.1 & -9.8 & -18.6 \\
20 & 0.7 & -24.1 & -89.3 & -13.7 & -42.6 \\
\hline 10 & -12.6 & -29.3 & -81.3 & -15.1 & -42.5 \\
5 & -11.9 & -33.5 & -75.6 & -11.5 & -34.4 \\
1 & -15.3 & -38.3 & -69.1 & -4.3 & -17.4 \\
0.1 & -13.6 & -42.4 & -59.3 & 14.2 & -11.1 \\
0.01 & -2.5 & -43.0 & -49.6 & 42.8 & -13.2 \\
\hline
\end{tabular}


Table S 6: Relative deviation [\%] of Flow Duration Curve (FDC) values between biascorrected (BC) Earth System Models (ESMs) and WATCH Forcing Data (WFD) in the period 1970-1999. $Q_{p t}=$ percentile of discharge.

\begin{tabular}{rrrrrr}
$Q_{p t}$ & GFDL & HadGEM & IPSL & MIROC & NorESM \\
\hline 99.99 & -3.4 & 36.5 & -107.0 & -93.4 & -70.7 \\
99.9 & -3.5 & 63.4 & -106.6 & -5.6 & -9.6 \\
99 & -66.8 & 55.6 & -101.1 & -33.6 & -68.8 \\
95 & -33.7 & 36.1 & -73.4 & 2.6 & -12.7 \\
90 & 1.4 & 26.3 & -27.6 & 8.6 & 14.5 \\
\hline 80 & 25.9 & 14.4 & -6.6 & -3.0 & 5.4 \\
70 & 31.8 & 10.5 & 7.8 & 2.3 & 18.6 \\
60 & 28.4 & 3.0 & 9.8 & 0.5 & 20.3 \\
50 & 29.5 & 0.4 & 9.1 & -0.4 & 17.1 \\
40 & 24.9 & -5.3 & 7.7 & -4.4 & 9.3 \\
30 & 15.3 & -12.1 & 1.9 & -11.5 & 2.8 \\
20 & -8.3 & -10.1 & -10.7 & -20.5 & -16.8 \\
\hline 10 & -7.5 & -15.3 & -5.2 & -15.1 & -17.9 \\
5 & -11.1 & -17.0 & 8.3 & -5.8 & -14.3 \\
1 & -10.1 & -20.9 & 45.3 & 20.2 & -1.2 \\
0.1 & 26.6 & -23.8 & 76.7 & 54.5 & 33.5 \\
0.01 & 77.1 & -24.7 & 142.0 & 66.1 & 41.4 \\
\hline
\end{tabular}


Table S 7: Relative deviation [\%] of Flow Duration Curve (FDC) values between uncorrected (UC) Regional Climate Models (RCMs) and WATCH Forcing Data (WFD) in the period 1970-1999. $Q_{p t}=$ percentile of discharge.

\begin{tabular}{rrrrrrrrrrr}
\multicolumn{10}{c}{ Model ID } \\
$Q_{p t}$ & 6 & 7 & 8 & 9 & 10 & 11 & 12 & 13 & 14 & 15 \\
\hline 99.99 & 611.8 & -107.0 & -106.1 & 1016.3 & 314.6 & 551.8 & 3048.1 & 736.0 & 433.3 & 2.2 \\
99.9 & 721.9 & -106.6 & -96.1 & 1103.3 & 476.5 & 820.7 & 3208.3 & 1184.7 & 478.4 & -1.4 \\
99 & 836.5 & -101.6 & -50.7 & 475.9 & 360.7 & 518.2 & 1104.2 & 803.1 & 377.2 & -33.5 \\
95 & 325.3 & -100.4 & -44.2 & 124.8 & 124.2 & 231.9 & 416.3 & 217.2 & 174.8 & -15.9 \\
90 & 217.2 & -100.0 & -44.5 & 69.6 & 101.2 & 159.0 & 296.0 & 121.8 & 128.9 & -21.1 \\
\hline 80 & 139.7 & -96.8 & -52.5 & 30.4 & 79.8 & 104.0 & 187.2 & 56.3 & 85.3 & -30.6 \\
70 & 122.4 & -92.7 & -50.5 & 27.5 & 74.6 & 96.4 & 169.0 & 42.0 & 76.6 & -30.7 \\
60 & 111.5 & -89.8 & -48.0 & 23.4 & 76.8 & 95.9 & 157.0 & 32.6 & 71.6 & -29.6 \\
50 & 120.8 & -87.9 & -43.7 & 29.8 & 88.1 & 104.7 & 157.7 & 26.5 & 74.1 & -28.2 \\
40 & 119.0 & -87.4 & -42.9 & 28.9 & 82.7 & 105.6 & 142.2 & 14.7 & 62.7 & -29.6 \\
30 & 71.4 & -86.4 & -48.2 & 14.4 & 63.3 & 79.1 & 115.3 & -8.1 & 42.1 & -39.3 \\
20 & 12.3 & -86.2 & -54.8 & -3.3 & 41.1 & 32.3 & 77.2 & -11.7 & 47.7 & -40.2 \\
\hline 10 & -2.9 & -74.3 & -45.9 & -9.6 & 35.1 & 9.0 & 56.1 & -1.5 & 32.8 & -15.0 \\
5 & -7.4 & -65.1 & -43.6 & -6.8 & 35.6 & 2.7 & 50.0 & 1.0 & 24.1 & -4.8 \\
1 & -5.5 & -53.6 & -44.4 & -3.2 & 32.5 & -1.3 & 46.1 & 6.2 & 21.6 & 12.1 \\
0.1 & 6.5 & -36.4 & -42.8 & 6.8 & 25.2 & 5.5 & 50.8 & 17.2 & 36.5 & 12.7 \\
0.01 & 15.8 & -15.6 & -41.6 & 17.3 & 31.9 & 16.8 & 60.7 & 31.1 & 36.0 & 21.7 \\
\hline
\end{tabular}


Table S 8: Relative deviation [\%] of Flow Duration Curve (FDC) values between biascorrected (BC) Regional Climate Models (RCMs) and WATCH Forcing Data (WFD) in the period 1970-1999. $Q_{p t}=$ percentile of discharge.

\begin{tabular}{rrrrrrrrrrr}
\multicolumn{10}{c}{ Model ID } \\
$Q_{p t}$ & 6 & 7 & 8 & 9 & 10 & 11 & 12 & 13 & 14 & 15 \\
\hline 99.99 & -101.4 & -107.0 & -106.9 & -106.9 & -106.4 & -106.9 & -3.4 & -106.8 & -107.0 & -106.8 \\
99.9 & -33.8 & -106.6 & -104.4 & -103.3 & -95.4 & -106.4 & -7.7 & -103.9 & -105.9 & -103.7 \\
99 & -10.8 & -101.6 & -67.4 & -41.7 & -74.2 & -50.2 & -71.8 & -73.1 & -67.9 & -68.5 \\
95 & -3.4 & -93.6 & -46.6 & -15.7 & -60.2 & -17.4 & -6.4 & -67.8 & -42.6 & -43.4 \\
90 & -7.4 & -86.8 & -32.5 & -15.7 & -42.5 & -5.3 & 0.1 & -51.0 & -12.2 & -34.3 \\
\hline 80 & -16.8 & -65.7 & -24.4 & -9.7 & -13.7 & -9.6 & -7.6 & -35.3 & -3.9 & -24.9 \\
70 & -14.0 & -46.3 & -10.7 & -1.6 & 1.4 & -5.4 & 2.0 & -23.1 & 4.2 & -15.9 \\
60 & -11.7 & -35.1 & -6.2 & 6.0 & 9.9 & 4.8 & 7.8 & -17.3 & 6.8 & -10.7 \\
50 & -1.8 & -20.1 & 0.3 & 16.7 & 15.9 & 9.3 & 12.3 & -6.4 & 9.8 & 1.9 \\
40 & 5.7 & -14.8 & 2.1 & 18.1 & 11.0 & 3.4 & 4.9 & -6.5 & 8.6 & 5.2 \\
30 & 10.1 & -30.4 & -6.9 & 0.4 & 2.7 & -7.7 & -11.1 & -18.0 & -1.5 & -6.6 \\
20 & -6.2 & -40.6 & -13.2 & -11.2 & -6.6 & -16.4 & -11.4 & -23.3 & -10.8 & -17.9 \\
\hline 10 & 8.9 & -16.3 & -0.9 & 11.0 & 9.9 & -1.1 & 3.3 & -9.0 & 1.0 & 7.1 \\
5 & 37.2 & 24.5 & 15.3 & 39.6 & 22.8 & 23.3 & 26.9 & 17.7 & 21.4 & 43.9 \\
1 & 140.5 & 120.3 & 53.0 & 109.5 & 52.4 & 116.0 & 98.4 & 86.2 & 67.5 & 146.8 \\
0.1 & 327.6 & 279.1 & 132.9 & 242.5 & 71.3 & 260.0 & 213.5 & 245.8 & 185.8 & 246.9 \\
0.01 & 656.9 & 593.0 & 146.7 & 411.2 & 100.4 & 418.2 & 233.8 & 313.5 & 217.3 & 401.0 \\
\hline
\end{tabular}




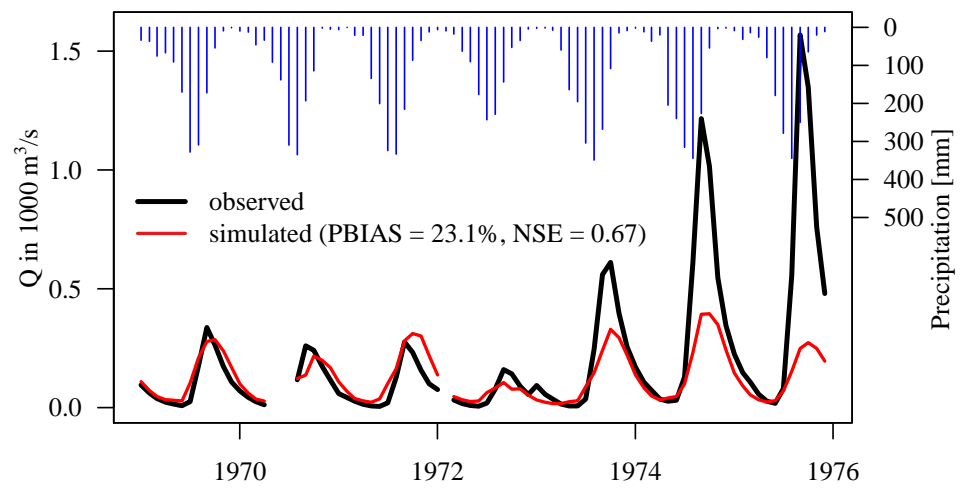

(a) downstream Tana

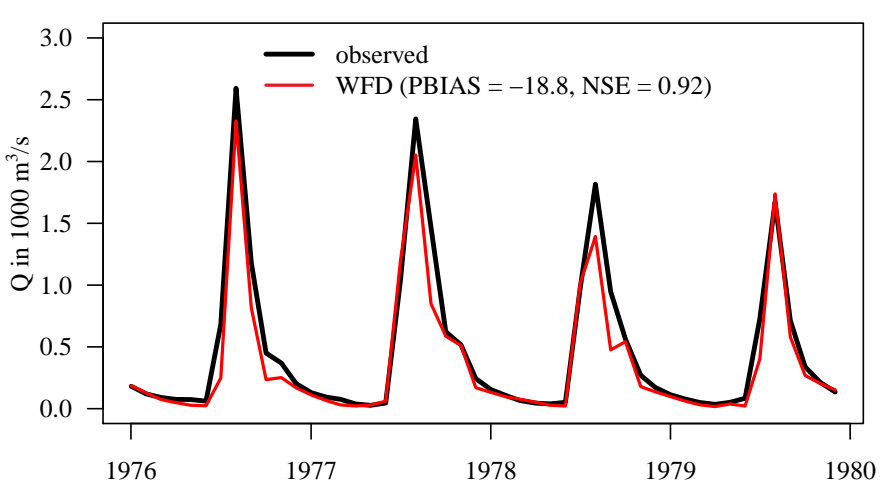

(b) Kessie

Figure S 1: Monthly observed and simulated discharges at gauges downstream Lake Tana and Kessie both upstream El Diem. Blue bars in top figure show monthly precipitation amounts. Y-axes indicate the year. 

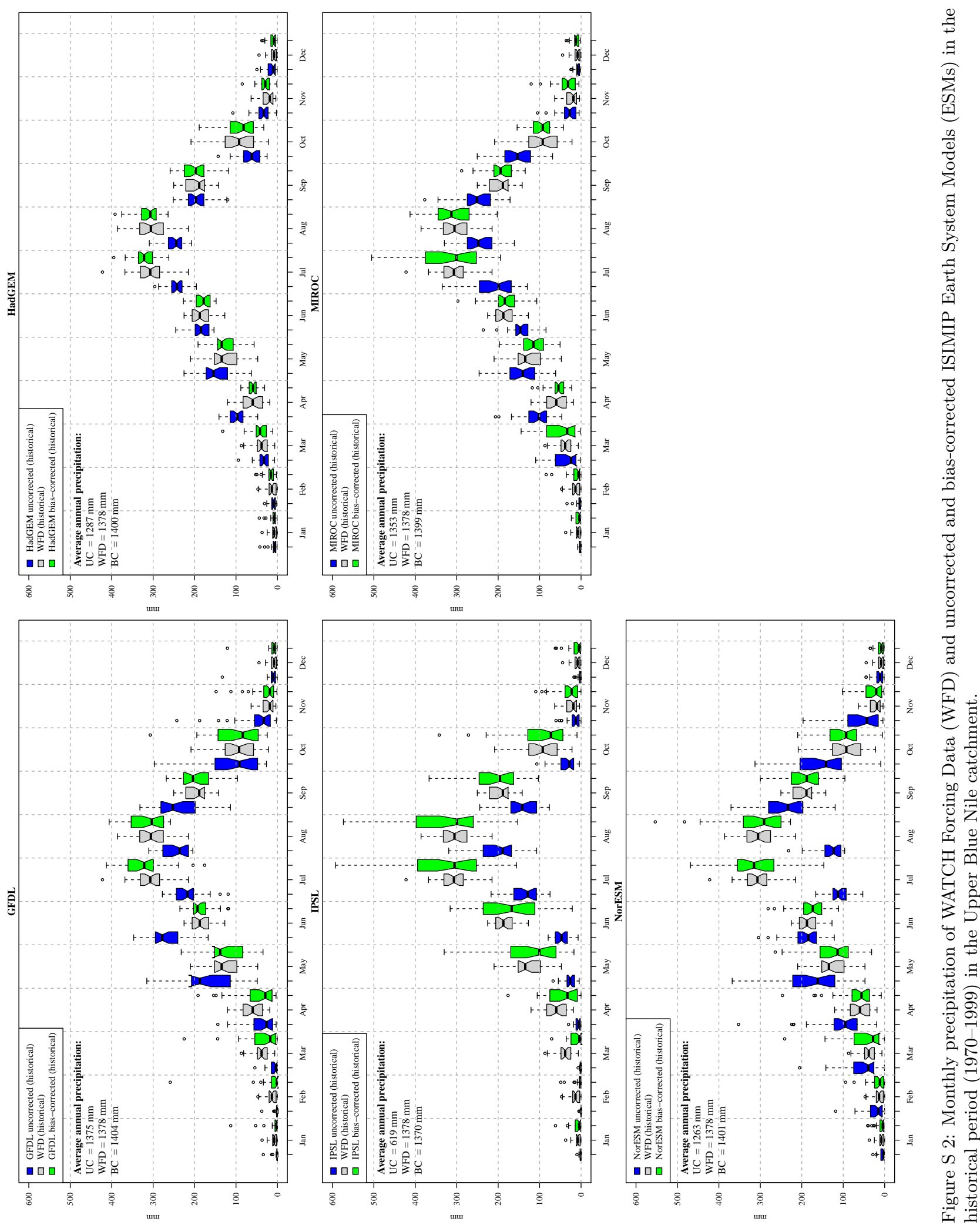

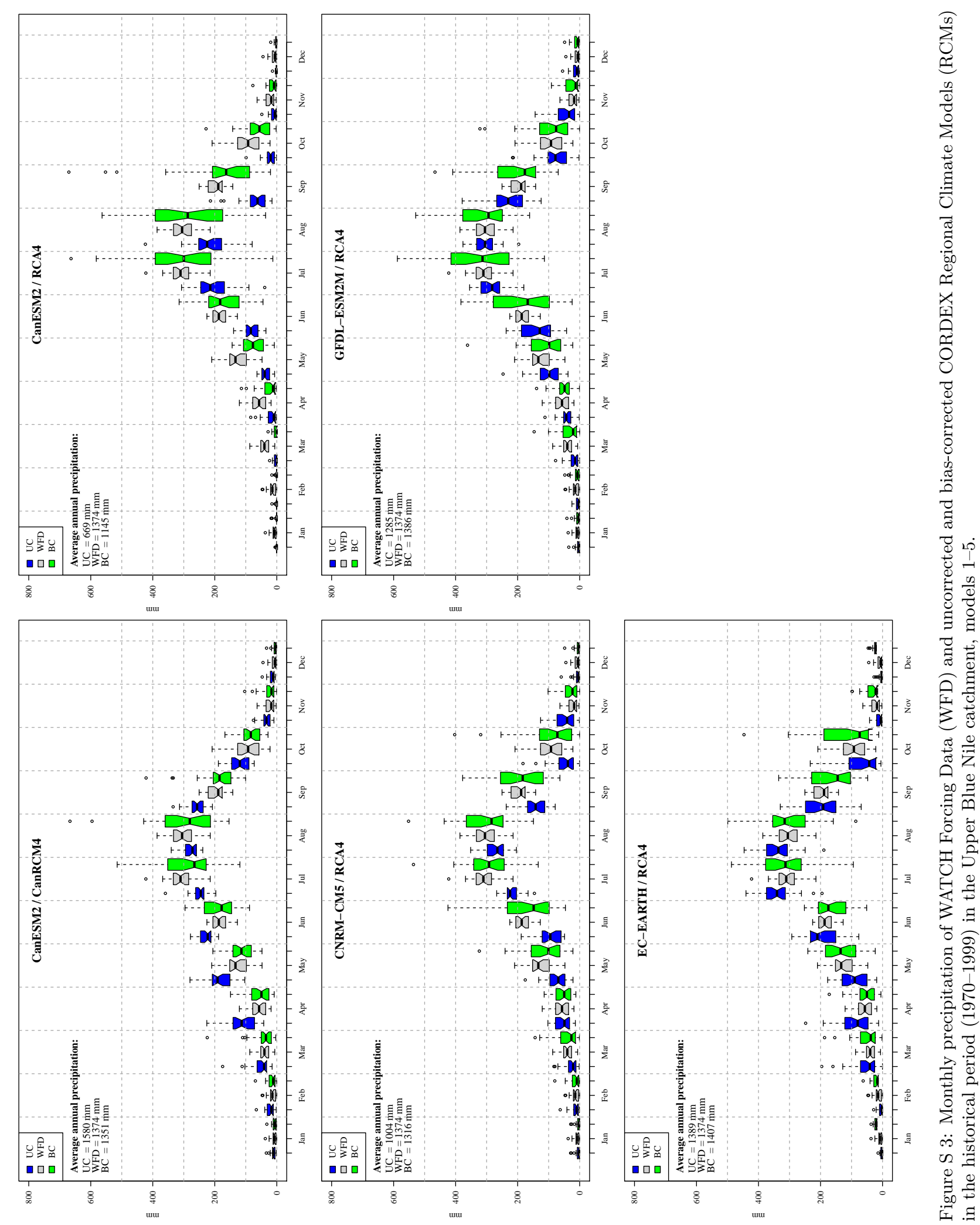

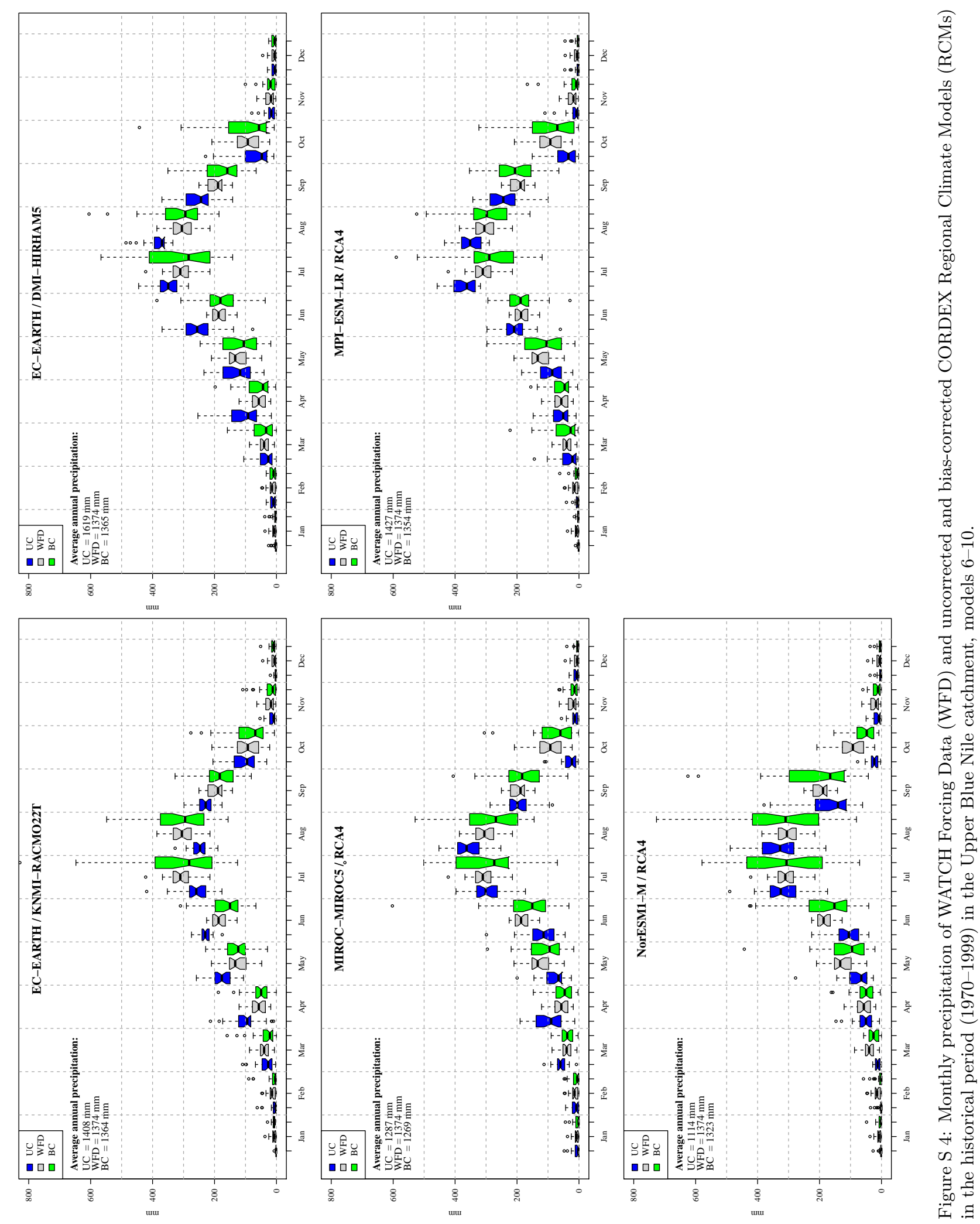


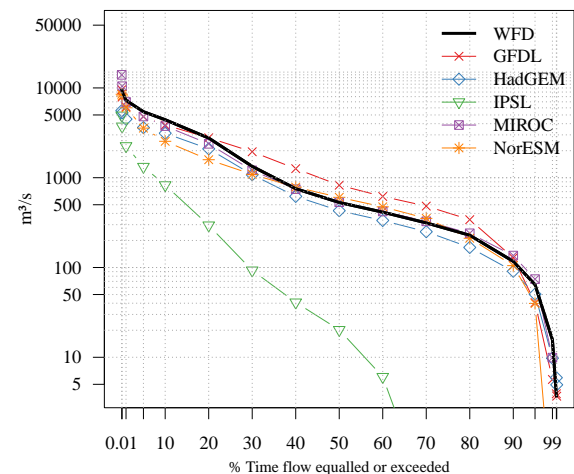

(a) ESMs (UC)

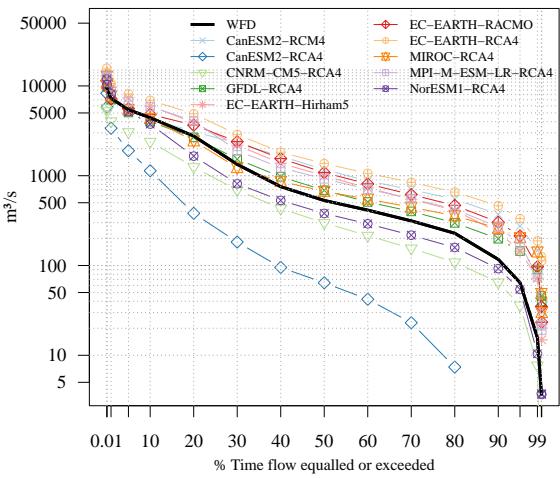

(c) RCMs (UC)

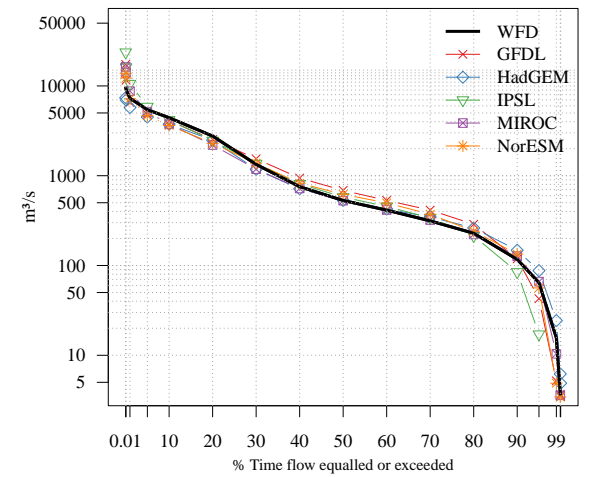

(b) ESMs (BC)

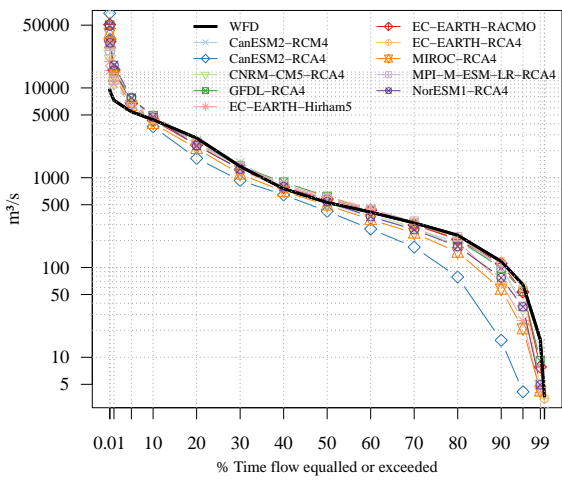

(d) $\mathrm{RCMs}(\mathrm{BC})$

Figure S 5: Flow Duration Curves (FDCs) of average daily discharge at gauge El Diem using WATCH Forcing Data (WFD), uncorrected (UC) and bias-corrected (BC) Earth System Model (ESM) and Regional Climate Model (RCM) climate input in the reference period (1970-1999). 

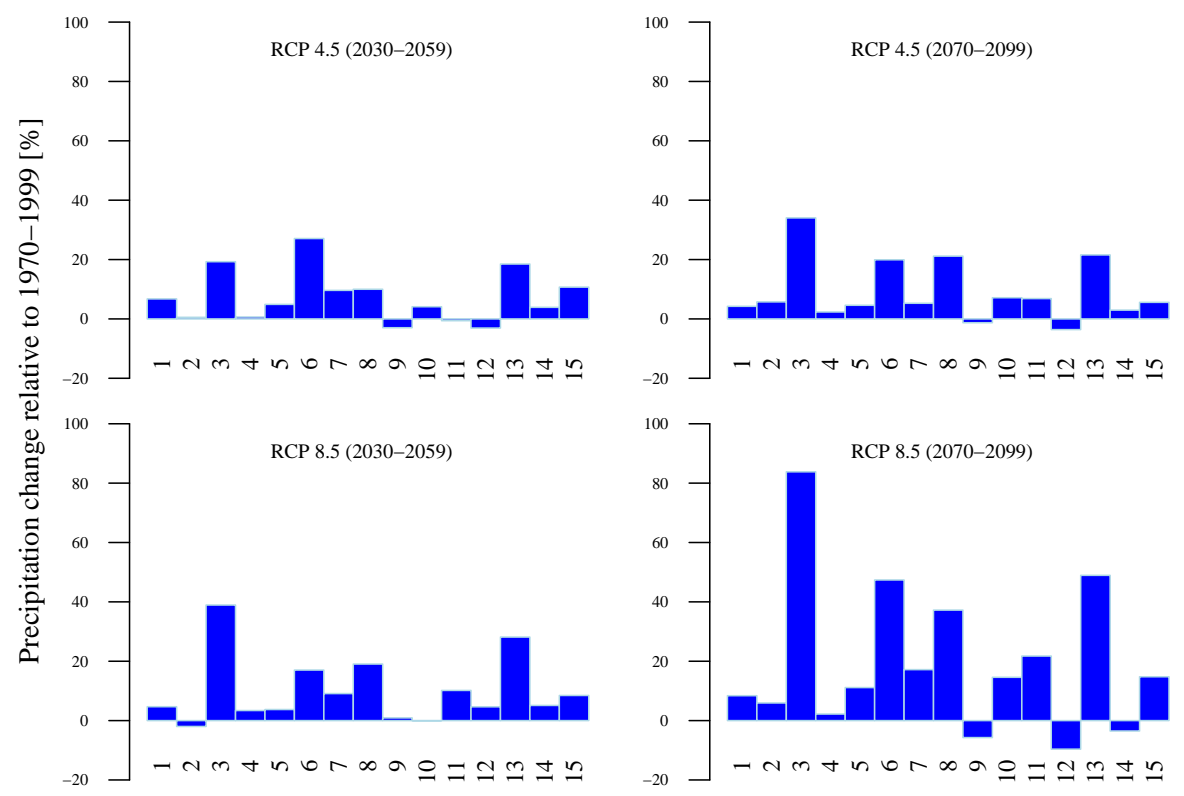

Models (1-15)

Figure S 6: Relative annual precipitation changes of uncorrected (UC) climate models in the Upper Blue Nile catchment

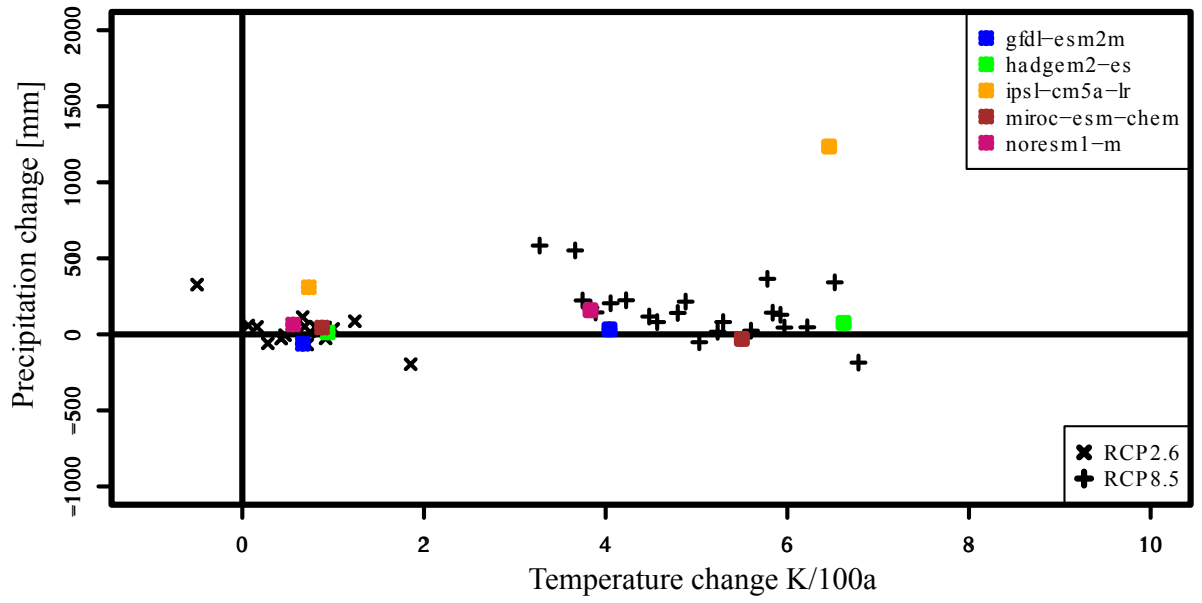

Figure S 7: Precipitation and temperature changes in the UBN catchment (CMIP5 ensemble). Earth System Models (ESMs) used in this study are highlighted in colours. 


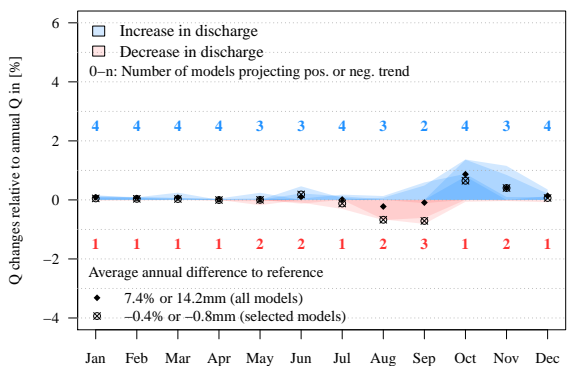

(a) ESMs, 2030-2059 (UC)

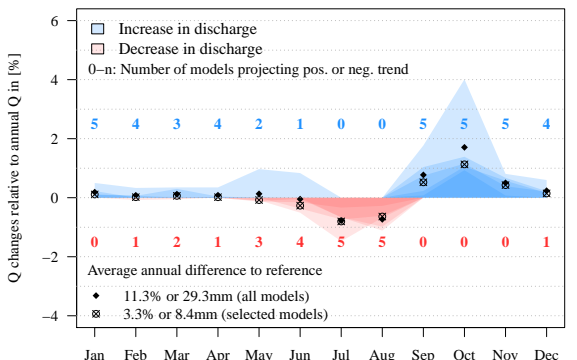

(c) ESMs, 2030-2059 (BC)

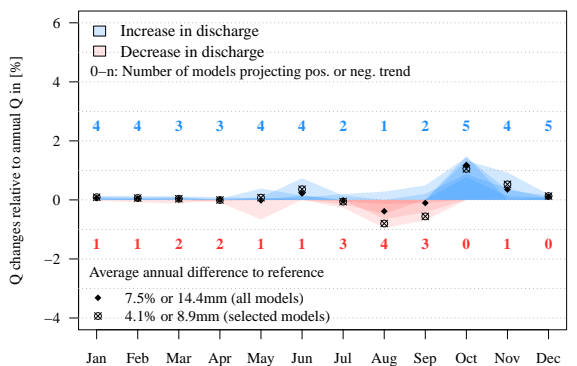

(b) ESMs, 2070-2099 (UC)

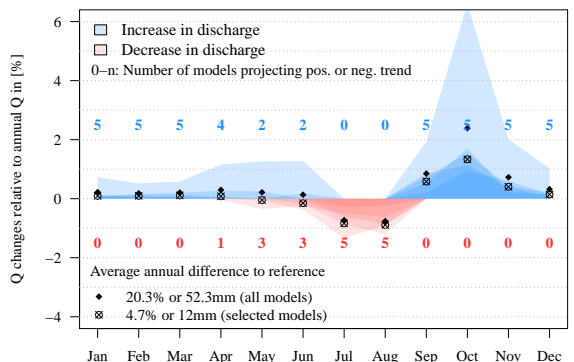

(d) ESMs, 2070-2099 (BC)

Figure S 8: Monthly discharge changes of uncorrected (UC) and bias-corrected (BC) Earth System Model (ESM) simulations in [\%] under RCP 4.5. Changes are relative to average annual discharge in the reference period (1970-1999) at gauge El Diem. 


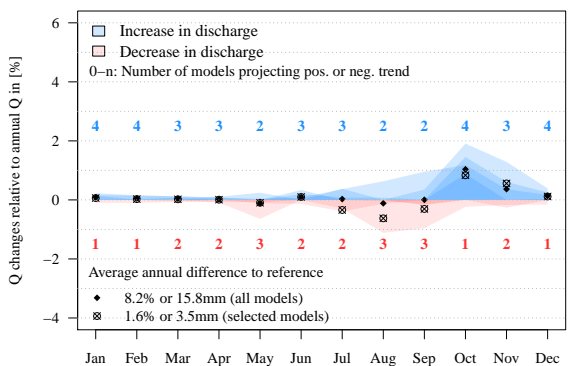

(a) ESMs, 2030-2059 (UC)

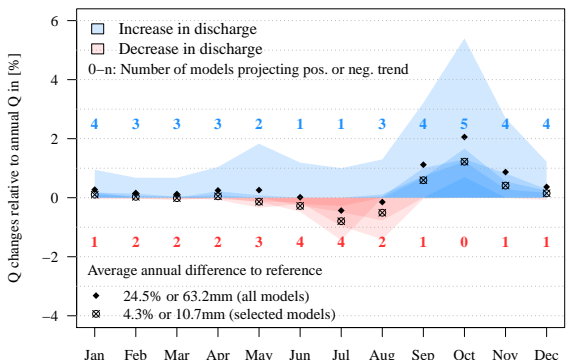

(c) ESMs, 2030-2059 (BC)

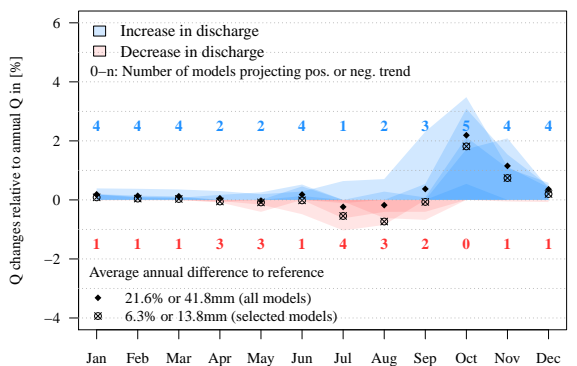

(b) ESMs, 2070-2099 (UC)

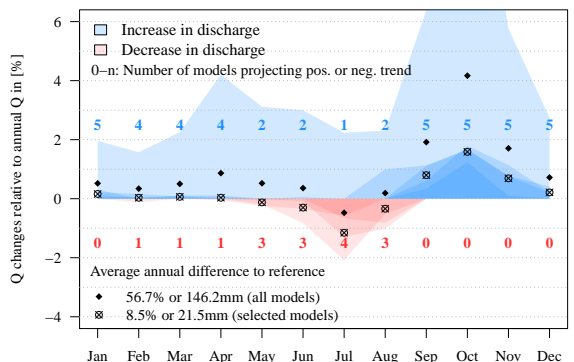

(d) ESMs, 2070-2099 (BC)

Figure S 9: Monthly discharge changes of uncorrected (UC) and bias-corrected (BC) ESM simulations in [\%] under RCP 8.5. Changes are relative to average annual discharge in the reference period (1970-1999) at gauge El Diem. 


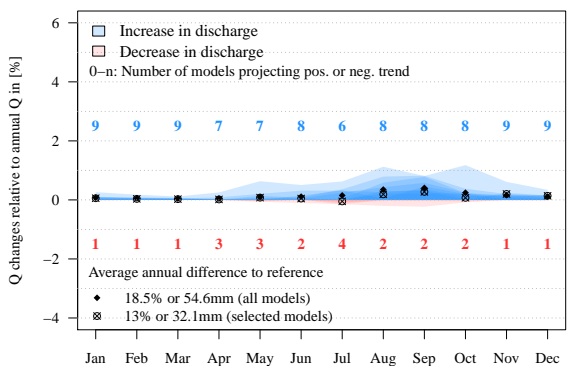

(a) RCMs, 2030-2059 (UC)

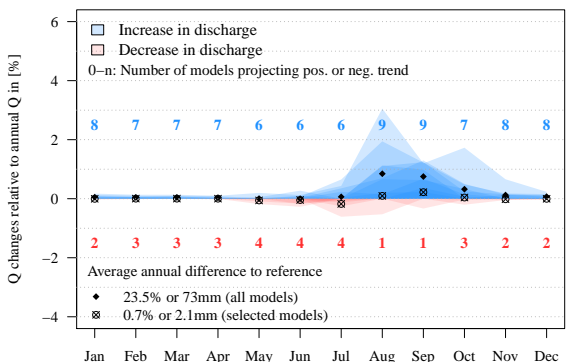

(c) RCMs, 2030-2059 (BC)

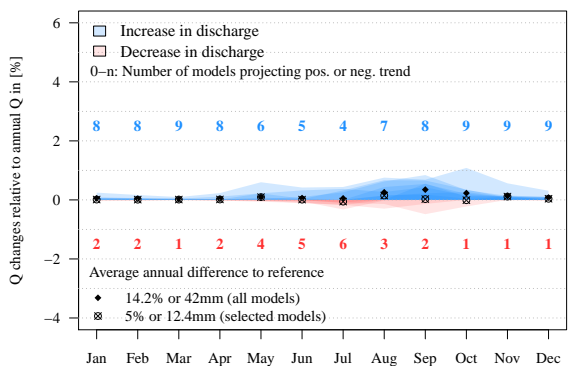

(b) RCMs, 2070-2099 (UC)

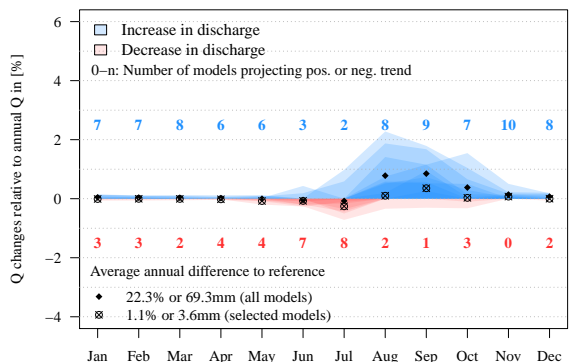

(d) RCMs, 2070-2099 (BC)

Figure S 10: Monthly discharge changes of uncorrected (UC) and bias-corrected (BC) RCM simulations in [mm] under RCP 4.5. Changes are relative to the reference period (1970-1999) at gauge El Diem. 


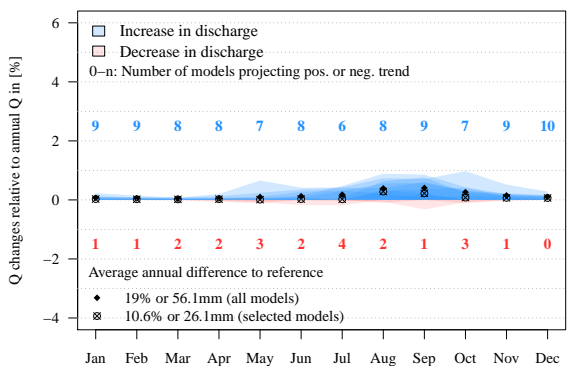

(a) RCMs, 2030-2059 (UC)

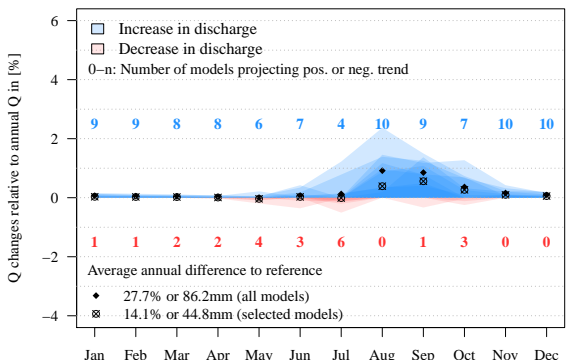

(c) RCMs, 2030-2059 (BC)

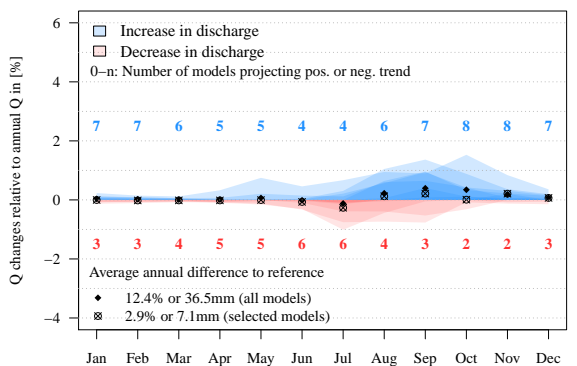

(b) RCMs, 2070-2099 (UC)

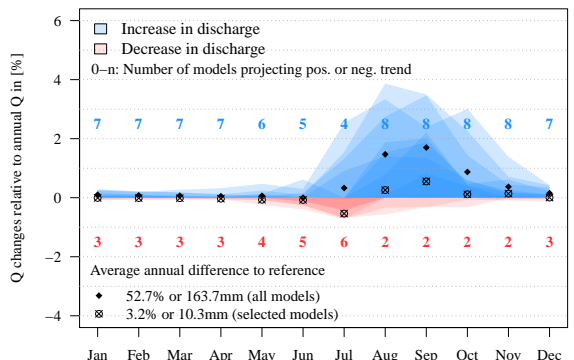

(d) RCMs, 2070-2099 (BC)

Figure S 11: Monthly discharge changes of uncorrected (UC) and bias-corrected (BC) RCM simulations in [mm] under RCP 8.5. Changes are relative to the reference period (1970-1999) at gauge El Diem. 